\title{
ARTICLE OPEN \\ Constructing oxide interfaces and heterostructures by atomic layer-by-layer laser molecular beam epitaxy
}

Qingyu Lei ${ }^{1}$, Maryam Golalikhani ${ }^{1}$, Bruce A. Davidson ${ }^{1,2}$, Guozhen Liu ${ }^{1}$, Darrell G. Schlom ${ }^{3,4}$, Qiao Qiao ${ }^{1,5}$, Yimei Zhu ${ }^{5}$, Ravini U. Chandrasena ${ }^{1}$, Weibing Yang ${ }^{1}$, Alexander X. Gray ${ }^{1}$, Elke Arenholz ${ }^{6}$, Andrew K. Farrar ${ }^{7}$, Dmitri A. Tenne ${ }^{7}, \mathrm{Minh} \mathrm{Hu}^{8}{ }^{8}$, Jiandong $\mathrm{Guo}^{8}$, Rakesh K. Singh ${ }^{9}$ and Xiaoxing $\mathrm{Xi}^{1}$

Advancements in nanoscale engineering of oxide interfaces and heterostructures have led to discoveries of emergent phenomena and new artificial materials. Combining the strengths of reactive molecular-beam epitaxy and pulsed-laser deposition, we show here, with examples of $\mathrm{Sr}_{1+x} \mathrm{Ti}_{1-x} \mathrm{O}_{3+\delta}$, Ruddlesden-Popper phase $\mathrm{La}_{n+1} \mathrm{Ni}_{n} \mathrm{O}_{3 n+1}(n=4)$, and $\mathrm{LaAl}_{1+y} \mathrm{O}_{3(1+0.5 y)} / \mathrm{SrTiO}_{3}$ interfaces, that atomic layer-by-layer laser molecular-beam epitaxy significantly advances the state of the art in constructing oxide materials with atomic layer precision and control over stoichiometry. With atomic layer-by-layer laser molecular-beam epitaxy we have produced conducting $\mathrm{LaAlO}_{3} / \mathrm{SrTiO}_{3}$ interfaces at high oxygen pressures that show no evidence of oxygen vacancies, a capability not accessible by existing techniques. The carrier density of the interfacial two-dimensional electron gas thus obtained agrees quantitatively with the electronic reconstruction mechanism.

npj Quantum Materials (2017)2:10; doi:10.1038/s41535-017-0015-x

\section{INTRODUCTION}

Technological advances in atomic-layer control during oxide film growth have enabled the discoveries of new phenomena and new functional materials, such as the two-dimensional (2D) electron gas at the $\mathrm{LaAlO}_{3} / \mathrm{SrTiO}_{3}$ interface, ${ }^{1,2}$ and asymmetric three-component ferroelectric superlattices. ${ }^{3,4}$ Reactive molecular-beam epitaxy (MBE) and pulsed-laser deposition (PLD) are the two most successful growth techniques for epitaxial heterostructures of complex oxides. PLD possesses experimental simplicity, low cost, and versatility in the materials to be deposited. ${ }^{5}$ Reactive MBE employing alternately-shuttered elemental sources (atomic layerby-layer MBE, or ALL-MBE) can control the cation stoichiometry precisely, thus producing oxide thin films of exceptional quality. ${ }^{6-8}$ There are, however, limitations in both techniques. Reactive MBE can use only source elements whose vapor pressure is sufficiently high, excluding a large fraction of $4 d$ and $5 d$ metals. In addition, ozone is needed to create a highly oxidizing environment while maintaining low-pressure MBE conditions, which increases the system complexity. On the other hand, conventional PLD using a compound target often results in cation off-stoichiometry in the films. ${ }^{9,10}$ In this paper we present an approach that combines the strengths of reactive MBE and PLD: atomic layer-by-layer laser MBE (ALL-Laser MBE) using separate oxide targets. Ablating alternately the targets of constituent oxides, for example $\mathrm{SrO}$ and $\mathrm{TiO}_{2}$, a SrTiO film can be grown one atomic layer at a time. Stoichiometry for both the cations and oxygen in the oxide films can be controlled. Although the idea of depositing atomic layers by PLD has been explored since the early days of laser MBE, ${ }^{11,12}$ we show that levels of stoichiometry control and crystalline perfection rivaling those of reactive $M B E$ can be achieved by ALL-Laser MBE. The technique is effective for both non-polar (such as $\mathrm{SrTiO}_{3}$ ) and polar materials, such as the Ruddlesden-Popper (RP) phase $\mathrm{La}_{n+1} \mathrm{Ni}_{n} \mathrm{O}_{3 n+1}$ with $n=4$. By growing $\mathrm{LaAlO}_{3}$ films on $\mathrm{SrTiO}_{3}$ substrates at an oxygen pressure of $37 \mathrm{mTorr}$, sufficiently high to alleviate oxygen deficiency in $\mathrm{SrTiO}_{3}$, we show that the properties of the 2D electron gas at the $\mathrm{LaAlO}_{3} / \mathrm{SrTiO}_{3}$ interface are in quantitative agreement with the electronic reconstruction mechanism.

\section{RESULTS}

The principle of ALL-Laser MBE is schematically illustrated in Fig. 1a. The key difference between ALL-Laser MBE and conventional PLD or laser MBE is the use of separate oxide targetsinstead of using a compound target of $\mathrm{SrTiO}_{3}$, targets of $\mathrm{SrO}$ and $\mathrm{TiO}_{2}$ are switched back and forth as they are alternately ablated by a UV laser beam. In conventional PLD or laser MBE using a compound target, all elements are ablated at once and the film grows unit cell by unit cell. In ALL-Laser MBE using separate targets, on the other hand, the film is constructed one atomic layer at a time. The number of laser pulses on each target for one atomic layer is around 100, allowing a stoichiometry control of about $1 \%$.

It has been a common practice to control the layer-by-layer growth of thin film by recording and analyzing in real time its reflection high-energy electron diffraction (RHEED) pattern. ${ }^{13,}{ }^{14}$ The intensity of the specularly-reflected RHEED spot is commonly used, which oscillates depending on the step edge density of the film. One oscillation period corresponds to the deposition of one unit cell layer in the unit cell-by-unit cell growth. ${ }^{15}$ Haeni et al.

\footnotetext{
${ }^{1}$ Department of Physics, Temple University, Philadelphia, PA 19122, USA; ${ }^{2}$ CNR-Istituto Officina dei Materiali, TASC National Laboratory, Trieste I-34146, Italy; ${ }^{3}$ Department of Materials Science and Engineering, Cornell University, Ithaca, NY 14853, USA; ${ }^{4}$ Kavli Institute at Cornell for Nanoscale Science, Ithaca, New York 14853 , USA; ${ }^{5}$ Department of Condensed Matter Physics and Materials Science, Brookhaven National Laboratory, Long Island, NY 11973, USA; ${ }^{6}$ Advanced Light Source, Lawrence Berkeley National Laboratory, Berkeley, CA 94720, USA; ${ }^{7}$ Department of Physics, Boise State University, Boise, ID 83725, USA; ${ }^{8}$ Beijing National Laboratory for Condensed Matter Physics and Institute of Physics, Chinese Academy of Sciences, Beijing 100190, P. R. China and ${ }^{9}$ School of Materials, Arizona State University, Tempe, AZ 85287, USA Correspondence: Xiaoxing Xi (xiaoxing@temple.edu)
}

Received: 8 November 2016 Revised: 1 January 2017 Accepted: 18 January 2017 Published online: 27 February 2017 

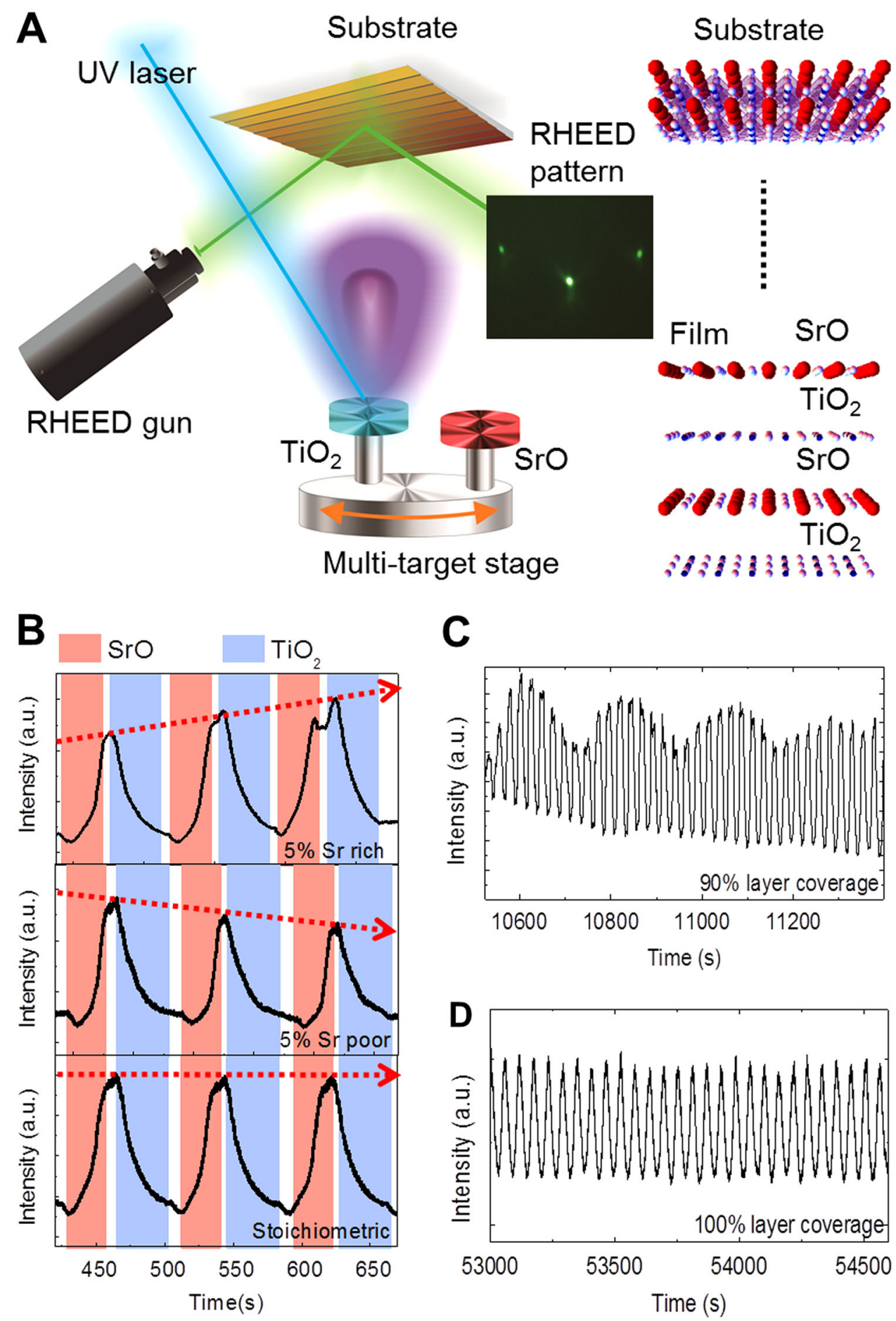

Fig. 1 a Schematic of the ALL-Laser MBE setup. SrO and $\mathrm{TiO}_{2}$ targets are alternately ablated by a UV laser beam while the RHEED pattern is being recorded and analyzed in real-time. The $\mathrm{SrTiO}_{3}$ film is constructed in an atomic layer-by-layer manner. b RHEED diffracted spot intensity oscillations during the atomic layer-by-layer growth of $\mathrm{SrTiO}_{3}$ films. The red and blue shaded areas represent the depositions of $\mathrm{SrO}$ and $\mathrm{TiO}_{2}$ layers, respectively. The white areas represent target switching when no ablation takes place. The red dotted lines indicate variations of the intensity for the cases of Sr-rich, Sr-poor, and stoichiometric deposition, respectively. c RHEED intensity beating when 0.9 monolayers of SrO and $\mathrm{TiO}_{2}$ are deposited during each target switching cycle. $\mathbf{d}$ Characteristic RHEED intensity oscillations during the growth of a stoichiometric $\mathrm{SrTiO}_{3}$ sample with full layer coverage

have found that the intensity of the diffracted spot can be used to control the growth of each atomic layer of $\mathrm{SrTiO}_{3}$ films in reactive MBE with alternately shuttered growth. ${ }^{7}$ In this work, we also use the diffracted spot intensity oscillation and our results confirm that the phenomenology identified by Haeni et al. also applies to ALL-Laser MBE. Figure $1 \mathrm{~b}$ shows the RHEED intensity oscillations as the targets of $\mathrm{SrO}$ and $\mathrm{TiO}_{2}$ are alternately ablated. Starting from a $\mathrm{TiO}_{2}$-terminated $\mathrm{SrTiO}_{3}$ substrate surface, the diffracted spot intensity increases to a maximum when one monolayer of $\mathrm{SrO}$ is deposited; it then decreases to a minimum when one monolayer of $\mathrm{TiO}_{2}$ is subsequently deposited. Furthermore, we have found that the specular spot also oscillates with the same 
period as the diffraction spot, albeit $180^{\circ}$ out of phase, if the Kikuchi lines caused by the diffused scattering of electrons do not overlap the specular spot (see Supplementary Materials).

The RHEED intensity depends on both the surface step edge density and the surface chemistry. When all elements of the film are delivered at the same time in the unit cell-by-unit cell growth, the chemistry information is averaged out and only the step edge density of the film is reflected in the RHEED intensity. For ALL-MBE or ALL-Laser MBE, the surface chemistry changes when different atomic layers are deposited sequentially; consequently both the step edge density and chemistry information can be observed. A detail discussion can be found in the Supplementary Materials.

In our experiment, the RHEED diffracted intensity oscillations along the $\mathrm{SrTiO}_{3}$ [110] azimuth were used to calibrate and control the film growth. As shown in Fig. $1 \mathrm{~b}, \mathrm{Sr} / \mathrm{Ti}>1$ leads to an increasing peak intensity and the appearance of a "double" peak, while $\mathrm{Sr} / \mathrm{Ti}<1$ leads to a reduced peak intensity; $\mathrm{Sr} / \mathrm{Ti}=1$ results in oscillation peaks with a constant intensity and shape. Furthermore, insufficient or excess pulses in each cycle cause beating of the RHEED intensity (Fig. 1c) while the intensity remains constant for $100 \%$ layer coverage (Fig. 1d). Using the RHEED intensity oscillation combined with the calibration of laser pulses per atomic layer obtained from the film thickness measurement, the cation stoichiometry in the films can be controlled to within $\pm 1 \%$.

The results of a series of $60 \mathrm{~nm}$ thick $\mathrm{Sr}_{1+x} \mathrm{Ti}_{1-x} \mathrm{O}_{3+\delta}$ films grown on $\mathrm{TiO}_{2}$-terminated (001) $\mathrm{SrTiO}_{3}$ substrates by ALL-Laser MBE are presented in Fig. 2. The $x$ values for the five films, determined by the Rutherford backscattering spectrometry (RBS) measurement, shown in Fig. 2a, are $0.13,0.05,-0.01,-0.07$, and -0.12 , respectively, with a measurement error of $\pm 5 \%$. They are consistent with the intended compositions $(x=0.10,0.05,0.00$, $-0.05,-0.10)$ controlled by the respective numbers of laser pulses. Figure $2 \mathrm{~b}$ shows X-ray diffraction (XRD) $\theta-2 \theta$ scans for the films around the $\mathrm{SrTiO}_{3} 002$ diffraction peak along with that of the $\mathrm{SrTiO}_{3}$ substrate. When the film is stoichiometric, the XRD spectrum cannot be distinguished from that of the single crystal $\mathrm{SrTiO}_{3}$ substrate. When the film is not stoichiometric, regardless of $\mathrm{Sr}$ rich or deficient, a diffraction peak from the film at a smaller angle than the substrate peak is seen, indicating a $c$-axis lattice expansion well established for homoepitaxial off-stoichiometric $\mathrm{SrTiO}_{3}{ }^{8,}{ }^{9}$ The $c$ lattice constant vs. $x$ is plotted for the films in Fig. 2c. Also plotted are data from films grown by reactive MBE for comparison. The results from the two techniques are in agreement with each other. In Fig. 2d, ultraviolet Raman spectra are presented for the five films as well as a stoichiometric film grown by reactive $\mathrm{MBE}^{16}$ and a single crystal $\mathrm{SrTiO}_{3}$ substrate. For bulk $\mathrm{SrTiO}_{3}$, the Raman spectrum shows only the second-order features, ${ }^{17}$ while the spectra of all the nonstoichiometric samples contain strong first-order Raman peaks, indicating the breakdown of the central inversion symmetry. The stoichiometric film shows spectra similar to those of the stoichiometric film grown by reactive MBE (broad and weak second-order Raman peaks). The results demonstrate that ALL-Laser MBE possesses the same excellent stoichiometry control as ALL-MBE.

The ALL-Laser MBE technique can not only grow thin films of stable phases like $\mathrm{SrTiO}_{3}$, but also fabricate layered materials that may not exist in nature. As an example, we have grown thin films of the RP phase $\mathrm{La}_{n+1} \mathrm{Ni}_{n} \mathrm{O}_{3 n+1}$ with $n=4$ on a $\mathrm{LaAlO}_{3}$ substrate. Figure $3 a$ shows a schematic of the $\mathrm{La}_{5} \mathrm{Ni}_{4} \mathrm{O}_{13}$ structure, in which 4 layers of $\mathrm{NiO}_{6}$ octahedra are sandwiched between the $\mathrm{NaCl}$-type $\mathrm{LaO}$ double layers. No successful deposition of phase-pure $\mathrm{La}_{n+1} \mathrm{Ni}_{n} \mathrm{O}_{3 n+1}$ thin films with $n>3$ has been reported in the literature, likely due to thermodynamic instability. To grow the film by ALL-Laser $\mathrm{MBE}, \mathrm{La}_{2} \mathrm{O}_{3}$ and $\mathrm{NiO}$ targets were ablated alternately to deposit the $\mathrm{LaO}$ and $\mathrm{NiO}_{2}$ atomic layers four times before an additional $\mathrm{LaO}$ layer was added. This is shown in Fig. $3 \mathrm{~b}$ by the RHEED intensity oscillations, in which the fourth LaO deposition in each period contains twice as many laser pulses as the other $\mathrm{LaO}$ layers. The XRD $\theta-2 \theta$ scan of the resultant 50 unit-cell $\mathrm{La}_{5} \mathrm{Ni}_{4} \mathrm{O}_{13}$ film is presented in Fig. $3 c$, showing the diffraction peaks of the $n=4$ RP phase from 0010-0028 without any impurity peaks or peak splitting. Figure $3 \mathrm{~d}$ is a cross-sectional scanning transmission electron microscope (STEM) high angle annular dark field (HAADF) image of the $\mathrm{La}_{5} \mathrm{Ni}_{4} \mathrm{O}_{13}$ film, showing an extra layer of LaO every 4 unit cells of $\mathrm{LaNiO}_{3}$. In Fig. 3e, atomic resolution elemental mapping using electron energy-loss spectroscopy (EELS) indicates a perfect match between the elemental distribution and the simultaneously taken HAADF image, verifying that the extra layer is indeed $\mathrm{LaO}$. Evidently, ablating from the targets of $\mathrm{La}_{2} \mathrm{O}_{3}$ and $\mathrm{NiO}$ is able to form the polar $\mathrm{LaO}$ and $\mathrm{NiO}_{2}$ layers to produce the $\mathrm{RP}$ structure. As shown by Sreedhar et al., the Ni next to the LaO rock salt layer changes its valence to maintain the charge neutrality in the RP phase. ${ }^{18}$
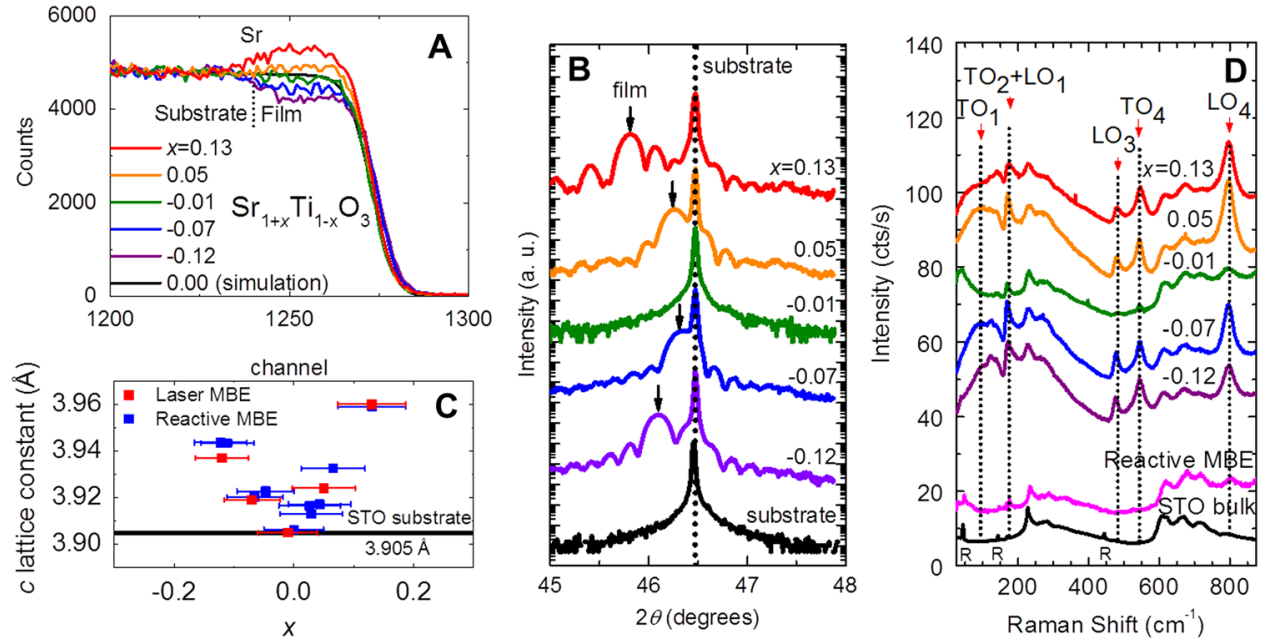

Fig. 2 a RBS spectra for five $60 \mathrm{~nm}$ thick $\mathrm{Sr}_{1+x} \mathrm{Ti}_{1-x} \mathrm{O}_{3+\delta}$ films grown by ALL-Laser MBE on $\mathrm{SrTiO}_{3}$ substrates. The compositions determined by RBS have an error of $\pm 5 \%$. b XRD $\theta-2 \theta$ scans of the films and a bare $\mathrm{SrTiO}_{3}$ substrate. The black dotted line represents the substrate peak position, and arrows point to film peaks. c The $c$ lattice constant (determined by the XRD measurement) vs. $x$ (determined by the RBS measurement) for the five films. The data from films grown by reactive MBE are included for comparison. $\mathbf{d}$ Ultraviolet Raman spectra $(\lambda=325 \mathrm{~nm}, T=10 \mathrm{~K})$ for the five films, a stoichiometric film grown by reactive $\mathrm{MBE}$, and a $\mathrm{SrTiO}_{3}$ single crystal 

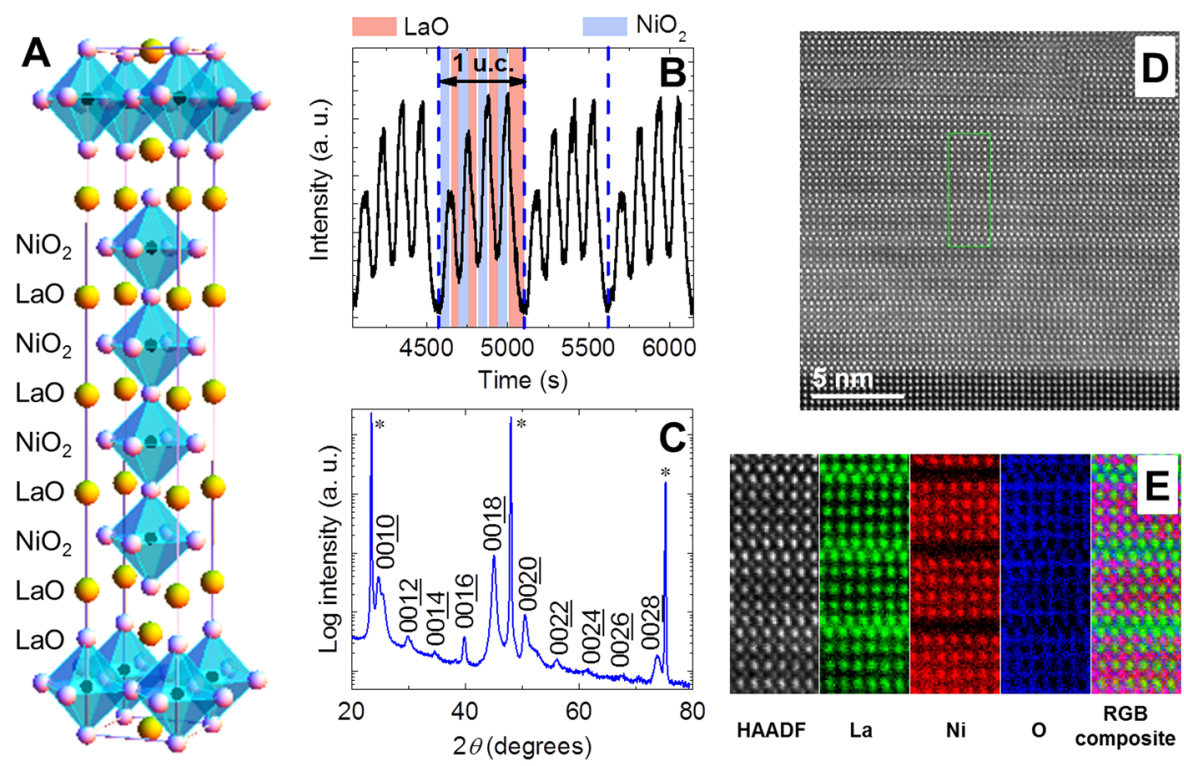

Fig. 3 a Schematic of the $\mathrm{La}_{n+1} \mathrm{Ni}_{n} \mathrm{O}_{3 n+1}$ structure with $n=4, \mathrm{La}_{5} \mathrm{Ni}_{4} \mathrm{O}_{13}$. b RHEED intensity oscillations during the growth of a $\mathrm{La}_{5} \mathrm{Ni}_{4} \mathrm{O}_{13}$ film. An extra $\mathrm{LaO}$ layer is inserted after every four pairs of $\mathrm{NiO}_{2}-\mathrm{LaO}$ layers are deposited. c XRD $\theta-2 \theta$ scan of the $\mathrm{La}_{5} \mathrm{Ni}_{4} \mathrm{O}_{13}$ film. $\mathbf{d}$ STEM HAADF image of the $\mathrm{La}_{5} \mathrm{Ni}_{4} \mathrm{O}_{13}$ film. e Simultaneously taken HAADF image and EELS elemental mapping for the area marked with a rectangular box in Fig. 3d

The ability to grow polar materials allows us to study the $2 \mathrm{D}$ electron gas at the $\mathrm{LaAlO}_{3} / \mathrm{SrTiO}_{3}$ interface using ALL-Laser MBE. Since its discovery ${ }^{2}$ several competing mechanisms have been proposed and intensely debated to explain the origin of the interfacial $2 \mathrm{D}$ electron gas, including electronic reconstruction, ${ }^{19}$ oxygen vacancies in the $\mathrm{SrTiO}_{3}$ substrate, ${ }^{20-22}$ and intermixing between the $\mathrm{LaAlO}_{3}$ film and the $\mathrm{SrTiO}_{3}$ substrate. ${ }^{23,}{ }^{24}$ According to the electronic reconstruction mechanism, because the atomic layers are charge neutral in $\mathrm{SrTiO}_{3}$ but charged in $\mathrm{LaAlO}_{3}$, a diverging electric potential is built up in a $\mathrm{LaAlO}_{3}$ film grown on a $\mathrm{TiO}_{2}$-terminated $\mathrm{SrTiO}_{3}$ substrate. This leads to the transfer of half of an electron from the $\mathrm{LaAlO}_{3}$ film surface to $\mathrm{SrTiO}_{3}$ when the $\mathrm{LaAlO}_{3}$ layer is thicker than 4 unit cells, creating a $2 \mathrm{D}$ electron gas at the interface with a sheet carrier density of $3.3 \times 10^{14} / \mathrm{cm}^{2}$ for sufficiently thick $\mathrm{LaAlO}_{3}$. A serious inconsistency with this mechanism is that the carrier densities reported experimentally are invariably lower than the expected value ${ }^{25,26}$ except under conditions where reduction of $\mathrm{SrTiO}_{3}$ substrate is suspected. ${ }^{20,} 21$ Oxygen vacancies in $\mathrm{SrTiO}_{3}$ are known to contribute to conductivity, but all reported conducting $\mathrm{LaAlO}_{3} / \mathrm{SrTiO}_{3}$ interfaces have been grown at low oxygen pressures ( $<10 \mathrm{mTorr})$, and annealing in oxygen is often required; ${ }^{1,19,27,28}$ higher oxygen pressures during the PLD growth result in insulating samples ${ }^{27}$ or 3D island growth. ${ }^{29}$ Low growth pressures can not only cause oxygen vacancies in $\mathrm{SrTiO}_{3}$, but can also enhance bombardment effect due to energetic species that may lead to $\mathrm{La}-\mathrm{Sr}$ intermixing at the interface. ${ }^{27}$ At present, there is no consensus on the origin of the $2 \mathrm{D}$ electron gas at the $\mathrm{LaAlO}_{3} / \mathrm{SrTiO}_{3}$ interface.

Because we grow the $\mathrm{LaAlO}_{3}$ film one atomic layer at a time, we were able to grow conducting $\mathrm{LaAlO}_{3} / \mathrm{SrTiO}_{3}$ interfaces at an oxygen pressure as high as $37 \mathrm{mTorr}$ with ALL-Laser MBE. This high oxygen pressure helps to prevent the possible oxygen reduction in $\mathrm{SrTiO}_{3}$, ensure that the $\mathrm{LaAlO}_{3}$ films are sufficiently oxygenated, and suppress the $\mathrm{La}-\mathrm{Sr}$ intermixing due to the bombardment effect. Furthermore, we grew $\mathrm{LaAlO}_{3}$ films of different cation stoichiometry, $\mathrm{LaAl}_{1+y} \mathrm{O}_{3(1+0.5 y)}$, as a way to test the electronic reconstruction hypothesis. As depicted by Sato et al., ${ }^{28}$ either Al vacancies or La vacancies in the off-stoichiometric films lead to oxygen vacancies in order to keep the charge neutrality. As a result, the charges on each layer depend on the stoichiometry: the charge on the $\left[\mathrm{LaO}_{1+y}\right]$ layer is $+(1-2 y)$ and the charge on the $\left[\mathrm{Al}_{1+y} \mathrm{O}_{2+0.5 y}\right]$ layer is $-(1-2 y)$. A similar modification of charges in each layer has been suggested for $\mathrm{LaAlO}_{3}$ films diluted with $\mathrm{SrTiO}_{3}{ }^{30}$ In the electronic reconstruction picture, instead of the charge transfer of half of an electron in the case of stoichiometric $\mathrm{LaAlO}_{3},(0.5-y)$ electrons will be transferred to resolve the polar discontinuity at the interface. The sheet carrier density depends linearly on $y$, i.e. $n_{s}=(1-2 y) \times 3.3 \times 10^{14} / \mathrm{cm}^{2}$ for sufficiently thick $\mathrm{LaAlO}_{3} .{ }^{28}$ For the 10 unit-cell $\mathrm{LaAlO}_{3}$ films used in this work, the dependence becomes $n_{s}=(1-2 y) \times 3.3 \times 10^{14} / \mathrm{cm}^{2}-1.6 \times 10^{14} / \mathrm{cm}^{2}$ $=(1.7-2 y \times 3.3) \times 10^{14} / \mathrm{cm}^{2}$. ${ }^{31}$

Starting from a $\mathrm{TiO}_{2}$-terminated $\mathrm{SrTiO}_{3}$ substrate, we grew 10 unit-cell-thick $\mathrm{LaAlO}_{3}$ films by alternately ablating $\mathrm{La}_{2} \mathrm{O}_{3}$ and $\mathrm{Al}_{2} \mathrm{O}_{3}$ targets under an oxygen pressure of 37 mTorr. The RHEED intensity oscillations during the growth of a stoichiometric film are shown in Fig. 4a. The RHEED intensity of the first LaO layer shows an irregular pattern, reflecting the state of surface chemistry, charge, and morphology during the transition from $\mathrm{TiO}_{2}$ to $\mathrm{LaO}$. The pattern becomes more regular in the subsequent layers. The reduction in the RHEED intensity from the substrate level is small throughout the growth of the 10 unit-cell stoichiometric $\mathrm{LaAlO}_{3}$ film. The $2 \mathrm{D}$ growth mode was maintained as confirmed by the sharp RHEED spots in Fig. $4 b$ for the 10 unitcell film. The atomic force microscopy (AFM) image for the film in Fig. $4 \mathrm{c}$ shows an atomically flat surface. To change the cation stoichiometry of the film, the number of laser pulses on the $\mathrm{Al}_{2} \mathrm{O}_{3}$ target for each $\mathrm{Al}_{1+y} \mathrm{O}_{2}$ layer was varied.

Because of the high oxygen pressure during the $\mathrm{LaAlO}_{3}$ growth, the samples were well oxygenated. This was proven by polarization-dependent X-ray absorption spectroscopy (XAS) measurements. Figure $4 d$ shows XAS spectra with different linear polarizations and the $\mathrm{Ti} L_{2,3} \mathrm{X}$-ray linear dichroism (XLD) signals are shown in Fig. $4 \mathrm{e}$ for different $\mathrm{LaAl}_{1+y} \mathrm{O}_{3(1+0.5 y)}$ stoichiometry. $\mathrm{No} \mathrm{Ti}^{3+}$ related features around $462 \mathrm{eV}$, characteristic of the oxygen deficient $\mathrm{LaAlO}_{3} / \mathrm{SrTiO}_{3}$ samples, ${ }^{32,}{ }^{33}$ are observed. Rather, the spectra are similar to the fully oxygenated samples. ${ }^{32,}{ }^{33}$ Figure $4 \mathrm{f}$ shows the Ti $L_{2,3} X$-ray magnetic circular dichroism (XMCD) signals obtained from opposite circularly polarized XAS spectra. Very small XMCD signals were observed, indicating very weak 

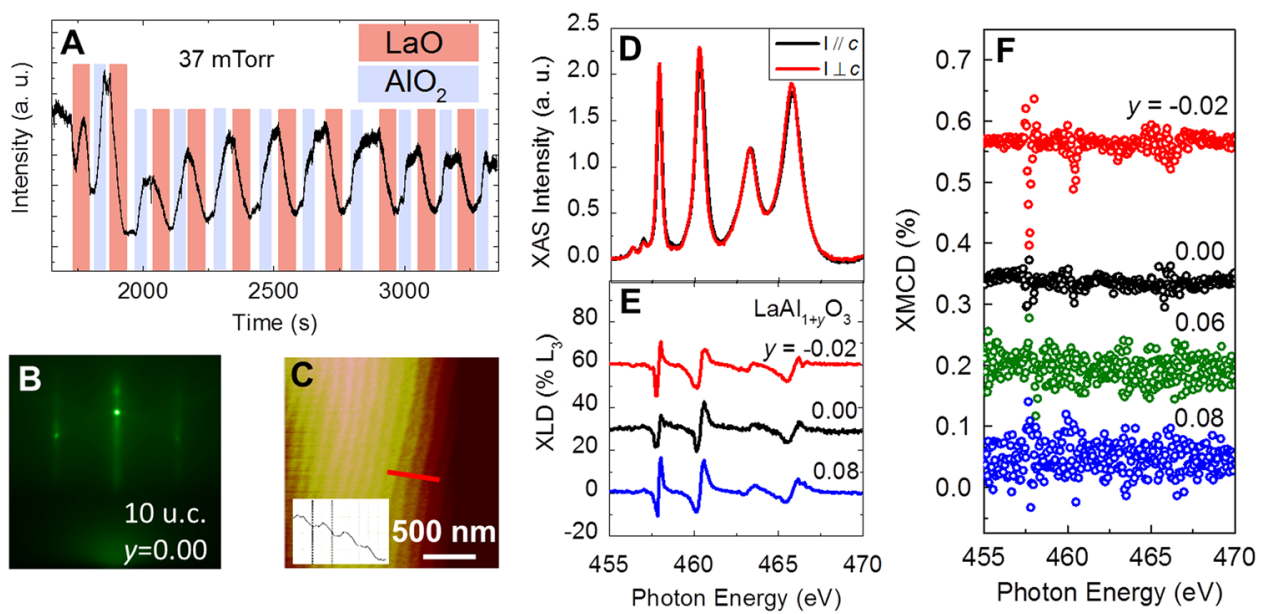

Fig. 4 a RHEED intensity oscillation during the growth of a $\mathrm{LaAlO}_{3}$ film on a $\mathrm{SrTiO}_{3}$ substrate at an oxygen pressure of $37 \mathrm{mTorr}$. $\mathbf{b}$ RHEED pattern after the growth of a 10 unit-cell stoichiometric $\mathrm{LaAlO}_{3}$ film. c AFM topographic images of the 10 unit-cell $\mathrm{LaAlO}_{3}$ film on $\mathrm{SrTiO}_{3}$. The root mean square (RMS) roughness of the film is $0.1 \mathrm{~nm}$. d Ti XAS spectra measured at $13 \mathrm{~K}$ with a polarization parallel (black curve) and perpendicular (red curve) to the sample normal for a 10 unit-cell stoichiometric $\mathrm{LaAlO}_{3}$ film on $\mathrm{SrTiO}_{3}$. e Ti $L_{2,3}$-edges XLD spectra for $3 \mathrm{LaAl}_{1+y} \mathrm{O}_{3(1+0.5 y)} / \mathrm{SrTiO}_{3}$ samples with $y=-0.02,0.00$, and 0.08 . f XMCD observed for the in-plane geometry for 4 LaAl $1+y \mathrm{O}_{3(1+0.5 y)}$ films on $\mathrm{SrTiO}_{3}$ with $y=-0.02,0.00,0.06$, and 0.08 . All samples were measured at a temperature of $13 \mathrm{~K}$ in a constant field of $\pm 0.3 \mathrm{~T}$

ferromagnetism. This again is consistent with the fully oxygen annealed $\mathrm{LaAlO}_{3} / \mathrm{SrTiO}_{3}$ samples. ${ }^{32,33}$

The temperature and stoichiometry dependences of the sheet resistance, sheet carrier density, and mobility are shown in Fig. 5a-f, respectively, for the 10 unit-cell thick $\mathrm{LaAl}_{1+y} \mathrm{O}_{3(1+0.5 y)}$ films. All of the films are conducting with sheet resistance around $10^{4} \Omega / \square$ at $300 \mathrm{~K}$, in contrast to insulating films grown by PLD from $\mathrm{LaAlO}_{3}$ compound targets at this oxygen pressure. ${ }^{27,}{ }^{34}$ Note that only the Al-rich $\mathrm{LaAl}_{1.08} \mathrm{O}_{3.12}$ film shows metallic behavior in the full temperature range, while all other films show low-temperature resistivity upturns, consistent with earlier reports of stoichiometry dependence. $^{35}, 36$ The low temperature upturn has a $-\ln T$ dependence characteristic of the Kondo effect. ${ }^{37}$ This may be attributed to the inevitable defects at the $\mathrm{LaAlO}_{3} / \mathrm{SrTiO}_{3}$ interface, consistent with the weak magnetism shown by Fig. 4f. The black dashed line in Fig. $5 \mathrm{~d}$ represents the threshold normal-state sheet resistance $h / 4 e^{2}$, or $6.5 \mathrm{k} \Omega / \square$ for a superconductor-insulator transition. ${ }^{38}$ Only the Al-rich samples have normal-state sheet resistance below the dashed line; these might thus exhibit superconductivity. The sheet carrier density is around $10^{14} / \mathrm{cm}^{2}$ for all the samples, close to the expected value of $1.7 \times 10^{14} / \mathrm{cm}^{2}$. The sample with a higher sheet carrier density shows a lower mobility, in agreement with previous reports. ${ }^{26}$

\section{DISCUSSION}

Thin film growth can overcome the bulk equilibrium thermodynamic limit in stabilizing new phases via various kinetic mechanisms $^{39}$ and elastic effects. ${ }^{40}$ By combining the strengths of reactive MBE and PLD, ALL-Laser MBE allows us to push the boundary further. Unlike stable phase materials $\mathrm{SrTiO}_{3}$ and $\mathrm{LaAlO}_{3}$, $\mathrm{La}_{5} \mathrm{Ni}_{4} \mathrm{O}_{13}$, the RP phase with $n=4$, has never been reported in the literature. Thermodynamic calculations indicate that the stability of higher- $n$-value RP phases requires higher oxygen pressure at higher temperature. ${ }^{41}$ ALL-Laser MBE makes the atomic layer-bylayer growth at high oxygen pressures possible, leading to the successful stabilization of $\mathrm{La}_{5} \mathrm{Ni}_{4} \mathrm{O}_{13}$. How far ALL-Laser MBE can stretch the thermodynamic limit is a question to be explored. For example, we have not been able to grow thin film of $\mathrm{BaMnO}_{3}$, which is not a thermodynamically stable phase in the bulk form.

The versatility of ALL-Laser MBE is demonstrated here by three very different examples: non-polar film on non-polar substrate (homoepitaxy of $\mathrm{SrTiO}_{3}$ ), polar film on polar substrate $\left(\mathrm{La}_{5} \mathrm{Ni}_{4} \mathrm{O}_{13}\right.$ on $\left.\mathrm{LaAlO}_{3}\right)$, and polar film on non-polar substrate $\left(\mathrm{LaAlO}_{3}\right.$ on $\left.\mathrm{SrTiO}_{3}\right)$. Laser ablation from oxide targets in oxygen atmosphere generates various neutrals, ions, and molecules in a propagating plume. ${ }^{42}$ Understanding how these species successfully react to form neutral (e.g., $\mathrm{SrO}$ and $\left.\mathrm{TiO}_{2}\right)$ or charged [e.g., $(\mathrm{LaO})^{+}$and $\left(\mathrm{AlO}_{2}\right)^{-}$] atomic layers in building up the oxide thin films and heterostructures in ALL-Laser MBE warrants theoretical investigations.

The central result of our work on the $\mathrm{LaAlO}_{3} / \mathrm{SrTiO}_{3}$ interface is the dependence of sheet carrier density on the stoichiometry of $\mathrm{LaAl}_{1+y} \mathrm{O}_{3(1+0.5 y)}$ shown in Fig. 5e. The black dashed line represents $n_{s}=(1.7-2 y \times 3.3) \times 10^{14} / \mathrm{cm}^{2}$, which is expected by the electronic reconstruction hypothesis for the 10 unit-cell films. ${ }^{31}$ The red squares, which denote $300 \mathrm{~K}$ sheet carrier density, overlap with the dashed line. No additional mechanism is employed to explain our data, and we cannot produce the linear dependence on the $\mathrm{LaAl}_{1+y} \mathrm{O}_{3(1+0.5 y)}$ stoichiometry quantitatively using either oxygen vacancies or intermixing mechanisms without invoking remarkable coincidence. The quantitative agreement between our experimental result and the theoretical prediction provides a strong support to the electronic reconstruction mechanism as responsible for the $2 \mathrm{D}$ electron gas at the $\mathrm{LaAlO}_{3} / \mathrm{SrTiO}_{3}$ interface.

The key differences between our result and the previous reports are the high oxygen pressure during the film growth and the high film crystallinity as demonstrated by Fig. 4 . We argue that the high oxygen pressure suppresses the likelihood of oxygen vacancies in $\mathrm{SrTiO}_{3}$. Well oxygenated samples produced during film growth can avoid possible defects when sufficient oxygen is provided only after the growth by annealing. Although ALL-Laser MBE may not prevent the possible thermodynamics-driven $\mathrm{SrTiO}_{3}-\mathrm{LaAlO}_{3}$ interdiffusion, the high oxygen pressure reduces the intermixing due to the bombardment effect. The $c$ lattice constant of a stoichiometric $\mathrm{LaAlO}_{3}$ film in this work was small (3.71 $\AA$ ), consistent with the expected value based on the Poisson ratio for a coherently strained film on $\mathrm{SrTiO}_{3}$, indicating a high structural perfection. Previous PLD studies on the stoichiometry dependence varied the laser energy density and oxygen pressure to change the $\mathrm{LaAlO}_{3}$ composition. ${ }^{28,}{ }^{36}$ In our study the films were grown under the same conditions, therefore, the stoichiometry dependence observed is free from the effects of varying deposition conditions. 

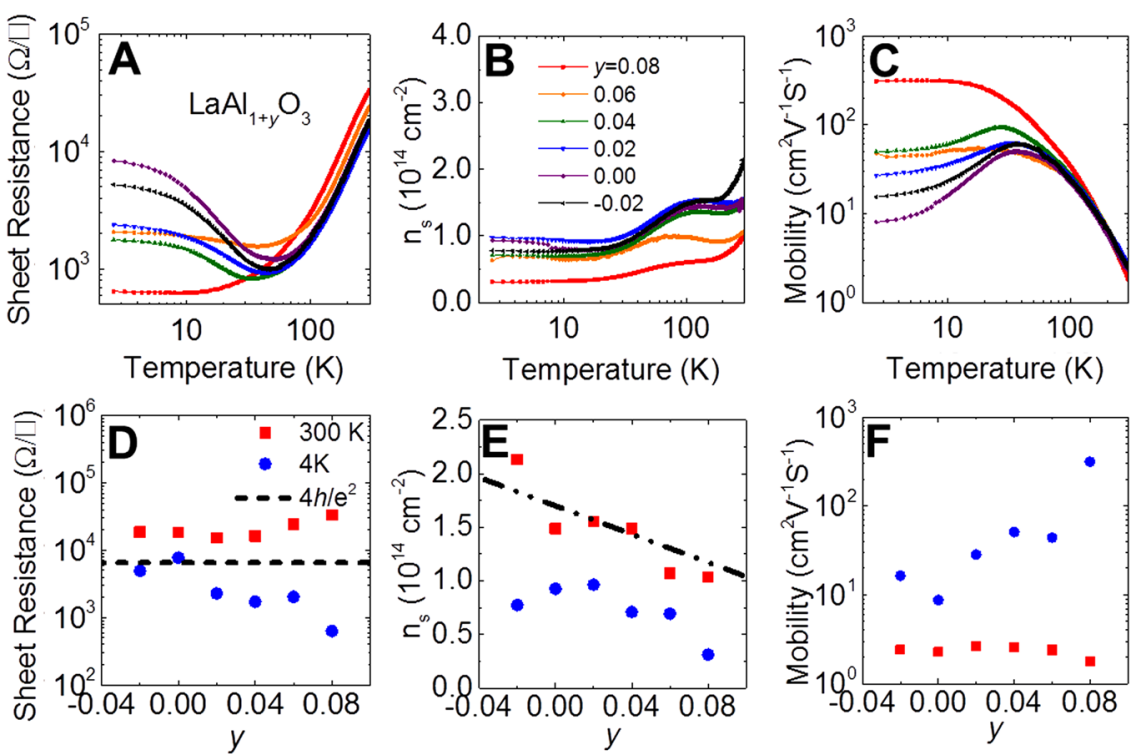

Fig. 5 a Sheet resistance, $\mathbf{b}$ carrier density, and $\mathbf{c}$ hall mobility as functions of temperature for a series of 10 unit-cell LaAl $1+y \mathrm{O}_{3(1+0.5 y)}$ films with different $y$ values. $\mathbf{d}$ Sheet resistance, e sheet carrier density, and $\mathbf{f}$ hall mobility as functions of film stoichiometry at $300 \mathrm{~K}$ (red squares) and $4 \mathrm{~K}$ (blue dots). The dashed line in $\mathbf{d}$ is the quantum resistance limit $h / 4 \mathrm{e}^{2} .{ }^{38}$ The dashed line in e indicates the theoretical value of sheet carrier density for 10 unit-cell films with different stoichiometry under the assumption of pure electronic reconstruction. ${ }^{31}$

As ALL-Laser MBE combines the atomic layer-by-layer growth of reactive MBE with PLD's versatility and compatibility with a broad range of oxygen pressures, it also inherits other characteristics of PLD such as the high kinetic energy of the ablated species, the impact of which needs to be studied. Nevertheless, ALL-Laser MBE is a significant addition to the toolbox of researchers aiming for the highest quality, new physics, new materials, and new functionalities in oxide thin films and heterostructures.

\section{METHODS}

The (001) $\mathrm{SrTiO}_{3}$ substrates used in this work were treated following the receipe in ref. 43 to produce atomically flat $\mathrm{TiO}_{2}$-terminated surface with one unit-cell-high steps. The (001) $\mathrm{LaAlO}_{3}$ substrates used in this work were treated following the receipe in ref. 44 to produce atomically flat $\mathrm{AlO}_{2}-$ terminated surface with one unit-cell-high steps.

A KrF excimer laser $(\lambda=248 \mathrm{~nm}$, pulse duration $25 \mathrm{~ns})$ was used with repetition rate ranging from 1 to $30 \mathrm{~Hz}$. For the growth of $\mathrm{SrTiO}_{3}$, a single crystal $\mathrm{SrO}$ target and a ceramic $\mathrm{TiO}_{2}$ target were used. The oxygen pressure during the growth was $1 \times 10^{-6} \mathrm{Torr}$ and the substrate temperature was $760{ }^{\circ} \mathrm{C}$. With a laser energy density of $0.9 \mathrm{~J} / \mathrm{cm}^{2}$, the number of laser pulses was about 100 for each SrO layer and 80 for each $\mathrm{TiO}_{2}$ layer in the growth of stoichiometric $\mathrm{SrTiO}_{3}$ film. The offstoichiometric films $\mathrm{Sr}_{1+x} \mathrm{Ti}_{1-x} \mathrm{O}_{3}$ were created by supplying $100 x \%$ more $\mathrm{SrO}$ and $100 x \%$ less $\mathrm{TiO}_{2}$ in each layer. After the deposition, the films were cooled to room temperature in oxygen at the same pressure as during the growth. For the growth of $\mathrm{La}_{5} \mathrm{Ni}_{4} \mathrm{O}_{13}$, ceramic targets of $\mathrm{La}_{2} \mathrm{O}_{3}$ and $\mathrm{NiO}$ were used. The oxygen pressure during the growth was $37 \mathrm{mTorr}$ and the substrate temperature was $600^{\circ} \mathrm{C}$. With the laser energy densities of $0.7 \mathrm{~J} / \mathrm{cm}^{2}$ for $\mathrm{LaO}$ and $0.9 \mathrm{~J} / \mathrm{cm}^{2}$ for $\mathrm{NiO}$, the number of laser pulses was 75 for each $\mathrm{LaO}$ layer and 107 for each $\mathrm{NiO}_{2}$ layer. The sequence for the growth of each RP unit cell is $\mathrm{LaO}-\mathrm{NiO}_{2}-\mathrm{LaO}-\mathrm{NiO}_{2}-\mathrm{LaO}-\mathrm{NiO}_{2}-\mathrm{LaO}-\mathrm{NiO}_{2}-\mathrm{LaO}$, i. e., four $\mathrm{LaNiO}_{3}$ layers followed by one extra $\mathrm{LaO}$ layer. After the deposition, the films were cooled to room temperature at a oxygen pressure of $8 \times 10^{4} \mathrm{~Pa}$. For the growth of $\mathrm{LaAlO}_{3}$ film, ceramic targets of $\mathrm{La}_{2} \mathrm{O}_{3}$ and $\mathrm{Al}_{2} \mathrm{O}_{3}$ were used. The oxygen pressure during the growth was $37 \mathrm{mTorr}$ and the substrate temperature was $720^{\circ} \mathrm{C}$. The laser energy density was $1.0 \mathrm{~J} / \mathrm{cm}^{2}$ on the $\mathrm{La}_{2} \mathrm{O}_{3}$ target, requiring 75 laser pulses for each $\mathrm{LaO}$ layer, and $1.3 \mathrm{~J} / \mathrm{cm}^{2}$ on the $\mathrm{Al}_{2} \mathrm{O}_{3}$ target, requiring 91 laser pulses for each $\mathrm{AlO}_{2}$ layer. The offstoichiometric $\mathrm{LaAl}_{1+y} \mathrm{O}_{3(1+0.5 y)}$ films were created by supplying $100 \%$ more $\mathrm{Al}$ in each layer. After the deposition, the films were cooled to room temperature in oxygen at the same pressure as during the growth.

$\mathrm{SrO}$ and $\mathrm{La}_{2} \mathrm{O}_{3}$ are not stable at ambient atmosphere-they react with water to form hydroxides and $\mathrm{La}_{2} \mathrm{O}_{3}$ also absorbs $\mathrm{CO}_{2}$ from the air. The $\mathrm{SrO}$ and $\mathrm{La}_{2} \mathrm{O}_{3}$ targets were removed from the vacuum sealed packages from the manufacture in a glovebox with a dry nitrogen atmosphere, mounted on the target holders, and then transferred to the vacuum chamber via a load-lock system swiftly such that their exposure to the air was as short as possible.

The XRD and reflectivity measurements were performed using a Bruker D8 Discover system with $\mathrm{Cu} \mathrm{K}_{\mathrm{a}}$ radiation $(\lambda=1.5406 \AA ̊)$. The Leptos fitting software (Bruker AXS Inc.) was used to determine the out-of-plane lattice constant of the films from the $\theta-2 \theta$ scans (the substrate peak was used as the reference) and to determine the film thickness from the X-ray reflectivity (XRR) measurement.

Ultraviolet Raman spectroscopy measurements were performed in a backscattering geometry normal to the film surface using a Jobin Yvon T64000 triple spectrometer equipped with a liquid nitrogen cooled multichannel charge coupled device detector. Ultraviolet light $(325 \mathrm{~nm}$ line of the $\mathrm{He}-\mathrm{Cd}$ laser) was used for excitation. Maximum laser power density was $\sim 0.5 \mathrm{~W} / \mathrm{mm}^{2}$ at the sample surface, low enough to avoid any noticeable local heating of the sample. Spectra were recorded at $10 \mathrm{~K}$ using a variable temperature closed-cycle helium cryostat.

XLD and XMCD measurements were carried out at the elliptically polarized undulator beamline 4.0.2 of the Advanced Light Source, using the vector magnet end station and with an energy resolution of approximately $0.1 \mathrm{eV}^{45}$ Samples were cryogenically cooled to $13 \mathrm{~K}$. Average probing depth in the total electron yield XAS detection mode was estimated to be approximately $5 \mathrm{~nm}$, providing interface-sensitive information with minimal contribution from surface adsorbates. Measurements were carried out in near-grazing $\left(30^{\circ}\right)$ incidence geometry, enabling selective alignment of the X-ray electric field parallel to the $a b$-plane of the film for vertically-polarized light $(E \| a b)$, and almost parallel to the $c$-axis of the film for vertically polarized light $(E \| c)$. The XMCD spectra were obtained utilizing circularly polarized X-rays with and by alternating the direction of the applied magnetic field of $0.3 \mathrm{~T}$ between parallel and antiparallel directions with respect to the $\mathrm{X}$-ray helicity vector.

\section{ACKNOWLEDGEMENTS}

The authors thank Dr. S. L. Shi and Dr. F. Q. Huang of Shanghai Institute of Ceramics, Chinese Academy of Sciences for synthesizing the ceramic SrO target which was used when developing the ALL-laser MBE technique. We thank Dr. P. S. Risborough for the helpful discussions concerning the Kondo effect. We thank Dr. Ph. Ghosez for comments on the manuscript and providing us with the first-principles calculation data on the $\mathrm{LaAlO}_{3}$ thickness dependence. This material is based upon work supported by the U.S. Department of Energy, Office of Science, under Grant No. DESC0004764 (Q. Y. L. and X. X. X.). Raman studies at Boise State University have been supported by NSF under Grant No. DMR-1006136 (A.K.F. and D. A. T.) TEM study was 
supported by CCDM, an EFRC funded by U.S. DOE-BES, under award \#DE-SC0012575 (Q.Q.), by DOE-BES, Materials Science and Engineering, under Contract No. DESC0012704 (Y.Z.), and used resources of CFN at BNL, a U.S. DOE Office of Science Facility. A.X.G. acknowledges support from the U.S. Army Research Office, under Grant No. W911NF-15-1-0181. The Advanced Light Source is supported by the Director, Office of Science, Office of Basic Energy Sciences, of the U.S. Department of Energy under Contract No. DE-AC02-05CH11231.

\section{AUTHOR CONTRIBUTIONS}

X. X. X. conceived and designed the research. Q. Y. L., M. G., and G. Z. L. carried out the experiment to develop the ALL-Laser MBE technique. B. A. D. was responsible for the growth calibration using the split RHEED intensity peaks. Q. Y. L. grew and characterized the $\mathrm{Sr}_{1+x} \mathrm{Ti}_{1-x} \mathrm{O}_{3+\delta}$ and $\mathrm{LaAl}_{1+y} \mathrm{O}_{3(1+0.5 y)} / \mathrm{SrTiO}_{3}$ samples. M. G. grew and characterized the Ruddlesden-Popper phase $\mathrm{La}_{n+1} \mathrm{Ni}_{n} \mathrm{O}_{3 n+1}(n=4)$ samples. D. G. S. participated in the conceptual design of ALL-Laser MBE and was responsible for the MBE-grown comparison samples of $\mathrm{Sr}_{1+x} \mathrm{Ti}_{1-x} \mathrm{O}_{3+\delta}$. Q. Q. and Y. M. Z. performed the TEM measurement. R. U. C., W. B. Y., A. X. G., and E. A. performed the XLD and XMCD measurements. A. K. F. and D. A. T. performed the UV Raman measurement. M. H. H. and J. D. G. contributed to the modeling of the RHEED intensity oscillations. R. K. S. performed the early RBS measurements. Q. Y. L., X. X. X. wrote the manuscript with significant contributions from D. G. S., A. X. G., and B. A. D. as well as contributions from all other authors.

\section{COMPETING INTERESTS}

The authors declare no competing interests.

\section{REFERENCES}

1. Hwang, H. Y. et al. Emergent phenomena at oxide interfaces. Nature Mater 11 103-113 (2012).

2. Ohtomo, A. \& Hwang, H. Y. A high-mobility electron gas at the $\mathrm{LaAlO}_{3} / \mathrm{SrTiO}_{3}$ heterointerface. Nature 427, 423-426 (2004).

3. Lee, H. N., Christen, H. M., Chisholm, M. F., Rouleau, C. M. \& Lowndes, D. H. Strong polarization enhancement in asymmetric three-component ferroelectric superlattices. Nature 433, 395-399 (2005).

4. Warusawithana, M. P., Colla, E. V., Eckstein, J. N. \& Weissman, M. B. Artificial dielectric superlattices with broken inversion symmetry. Phys. Rev. Lett. 90, 036802 (2003).

5. Willmott, P. R. \& Huber, J. R. Pulsed laser vaporization and deposition. Rev. Mod. Phys. 72, 315-328 (2000).

6. Eckstein, J. N. \& Bozovic, I. High-temperature superconducting multilayers and heterostructures grown by atomic layer-by-layer molecular beam epitaxy. Annu. Rev. Mater. Sci. 25, 679-709 (1995).

7. Heani, J. H., Theis, C. D. \& Schlom, D. G. RHEED intensity oscillations for the stoichiometric growth of $\mathrm{SrTiO}_{3}$ thin films by reactive molecular beam epitaxy. $J$ Electroceram. 4, 385-391 (2000).

8. Brooks, C. M. et al. Growth of homoepitaxial $\mathrm{SrTiO}_{3}$ thin films by molecular-beam epitaxy. Appl. Phys. Lett. 94, 162905 (2009).

9. Ohnishi, T., Shibuya, K., Yamamoto, T. \& Lippmaa, M. Defects and transport in complex oxide thin films. J. Appl. Phys. 103, 103703 (2008).

10. Liu, G. Z., Lei, Q. Y. \& Xi, X. X. Stoichiometry of $\mathrm{SrTiO}_{3}$ films grown by pulsed laser deposition. Appl. Phys. Lett. 100, 202902 (2012).

11. Kanai, M., Kawai, T. \& Kawai, S. Atomic layer and unit cell layer growth of $(\mathrm{Ca}, \mathrm{Sr})$ $\mathrm{CuO}_{2}$ thin film by laser molecular beam epitaxy. Appl. Phys. Lett. 58, 771-773 (1991).

12. Herklotz, A. et al. Stoichiometry control of complex oxides by sequential pulsedlaser deposition from binary-oxide targets. Appl. Phys. Lett. 106, 131601 (2015).

13. Wood, C. E. C. RED intensity oscillations during MBE of GaAs. Surf. Sci. Lett 108, L441-L443 (1981).

14. Neave, J. H., Joyce, B. A., Dobson, P. J. \& Norton, N. Dynamics of film growth of GaAs by MBE from rheed observations. Appl. Phys. A 31, 1-8 (1983).

15. Clarke, S. \& Vvedensky, D. D. Origin of reflection high-energy electron-diffraction intensity oscillations during molecular-beam epitaxy: a computational modeling approach. Phys. Rev. Lett. 58, 2235-2238 (1987).

16. Tenne, D. A. et al. Ferroelectricity in nonstoichiometric $\mathrm{SrTiO}_{3}$ films studied by ultraviolet Raman spectroscopy. Appl. Phys. Lett. 97, 142901 (2010).

17. Nilsen, W. G. \& Skinner, J. G. Raman spectrum of strontium titanate. J. Chem. Phys. 48, 2240-2248 (1968)

18. Sreedhar, K. et al. Low-temperature electronic properties of the $\mathrm{La}_{n+1} \mathrm{Ni}_{n} \mathrm{O}_{3 n+1}$ $(n=2,3$, and $\infty)$ system: evidence for a crossover from fluctuating-valence to Fermi-liquid-like behavior. J. Solid State Chem. 110, 208-215 (1994).
19. Nakagawa, N., Hwang, H. Y. \& Muller, D. A. Why some interfaces cannot be sharp. Nature Mater 5, 204-209 (2006).

20. Kalabukhov, A. et al. Effect of oxygen vacancies in the $\mathrm{SrTiO}_{3}$ substrate on the electrical properties of the $\mathrm{LaAlO}_{3} / \mathrm{SrTiO}_{3}$ interface. Phys. Rev. B. 75, 121404(R) (2007).

21. Siemons, W. et al. Origin of charge density at $\mathrm{LaAlO}_{3}$ on $\mathrm{SrTiO}_{3}$ heterointerfaces: possibility of intrinsic doping. Phys. Rev. Lett. 98, 196802 (2007).

22. Yu, L. P. \& Zunger, A. A polarity-induced defect mechanism for conductivity and magnetism at polar-nonpolar oxide interfaces. Nature Commun 5, 5118 (2014).

23. Willmott, P. R. et al. Structural basis for the conducting interface between $\mathrm{LaAlO}_{3}$ and $\mathrm{SrTiO}_{3}$. Phys. Rev. Lett. 99, 155502 (2007).

24. Chambers, S. A. et al. Instability, intermixing and electronic structure at the epitaxial $\mathrm{LaAlO}_{3} / \mathrm{SrTiO}_{3}(001)$ heterojunction. Surf. Sci. Rep. 65, 317-352 (2010).

25. Joshua, A., Pecker, S., Ruhman, J., Altman, E. \& Ilani, S. A universal critical density underlying the physics of electrons at the $\mathrm{LaAlO}_{3} / \mathrm{SrTiO}_{3}$ interface. Nat. Commun. 3, 1129 (2012).

26. Xie, Y., Bell, C., Hikita, Y., Harashima, S. \& Hwang, H. Y. Enhancing electron mobility at the $\mathrm{LaAlO}_{3} / \mathrm{SrTiO}_{3}$ interface by surface control. Adv. Mater. 25, 4735-4738 (2013).

27. Kalabukhov, A. et al. Improved cationic stoichiometry and insulating behavior at the interface of $\mathrm{LaAlO}_{3} / \mathrm{SrTiO}_{3}$ formed at high oxygen pressure during pulsedlaser deposition. Europhys. Lett. 93, 37001 (2011).

28. Sato, H. K., Bell, C., Hikita, Y. \& Hwang, H. Y. Stoichiometry control of the electronic properties of the $\mathrm{LaAlO}_{3} / \mathrm{SrTiO}_{3}$ heterointerface. Appl. Phys. Lett. 102, 251602 (2013).

29. Ariando et al. Electronic phase separation at the $\mathrm{LaAlO}_{3} / \mathrm{SrTiO}_{3}$ interface. Nat Commun. 2, 188 (2011).

30. Reinle-Schmitt, M. L. et al. Tunable conductivity threshold at polar oxide interfaces. Nat. Commun. 3, 932 (2012).

31. Cancellieri, C. et al. Electrostriction at the $\mathrm{LaAlO}_{3} / \mathrm{SrTiO}_{3}$ interface. Phys. Rev. Lett. 107, 056102 (2011).

32. Salluzzo, M. et al. Orbital Reconstruction and the two-dimensional electron gas at the $\mathrm{LaAlO}_{3} / \mathrm{SrTiO}_{3}$ interface. Phys. Rev. Lett. 102, 166804 (2009).

33. Salluzzo, M. et al. Origin of interface magnetism in $\mathrm{BiMnO}_{3} / \mathrm{SrTiO}_{3}$ and $\mathrm{LaAlO}_{3} /$ $\mathrm{SrTiO}_{3}$ heterostructures. Phys. Rev. Lett. 111, 087204 (2013).

34. Choi, W. S. et al. Atomic layer engineering of perovskite oxides for chemically sharp heterointerfaces. Adv. Mater. 24, 6423-6428 (2012).

35. Warusawithana, M. P. et al. $\mathrm{LaAlO}_{3}$ stoichiometry is key to electron liquid formation at $\mathrm{LaAlO}_{3} / \mathrm{SrTiO}_{3}$ interfaces. Nat. Commun. 4, 2351 (2013).

36. Breckenfeld, E., Bronn, N., Mason, N. \& Martin, L. W. Tunability of conduction at the $\mathrm{LaAlO}_{3} / \mathrm{SrTiO}_{3}$ heterointerface: thickness and compositional studies. Appl. Phys. Lett. 105, 121610 (2014).

37. Brinkman, A. et al. Magnetic effects at the interface between nonmagnetic oxides. Nat. Mater. 6, 493-496 (2007).

38. Haviland, D. B., Liu, Y. \& Goldman, A. M. Onset of superconductivity in the twodimensional limit. Phys. Rev. Lett. 62, 2180-2183 (1989).

39. Zhang, Z. Y. \& Lagally, M. G. Atomistic processes in the early stages of thin-film growth. Science 276, 377-383 (1997).

40. Müller, P. \& Saúl, A. Elastic effects on surface physics. Surf. Sci. Rep. 54, 157-258 (2004).

41. Zinkevich, M. et al. Stability and thermodynamic functions of lanthanum nickelates. J. Alloys Compd. 438, 92-99 (2007).

42. Geohegan, D. B. Physics and diagnostics of laser ablation plume propagation for high- $T_{c}$ superconductor film growth. Thin Solid Films 220, 138-145 (1992).

43. Kawasaki, M. et al. Atomic control of the $\mathrm{SrTiO}_{3}$ crystal surface. Science 266 1540-1542 (1994).

44. Ohnishi, T. et al. A-site layer terminated perovskite substrate: $\mathrm{NdGaO}_{3}$. Appl. Phys. Lett. 74, 2531-2533 (1999).

45. Young, A. T. et al. A soft x-ray undulator beamline at the advanced light source with circular and variable linear polarization for the spectroscopy and microscopy of magnetic materials. Surf. Rev. Lett. 9, 549-554 (2002).

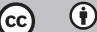

This work is licensed under a Creative Commons Attribution 4.0 International License. The images or other third party material in this article are included in the article's Creative Commons license, unless indicated otherwise in the credit line; if the material is not included under the Creative Commons license, users will need to obtain permission from the license holder to reproduce the material. To view a copy of this license, visit http://creativecommons.org/licenses/by/ $4.0 /$

(c) The Author(s) 2017

Supplementary Information accompanies the paper on the npj Quantum Materials website (doi:10.1038/s41535-017-0015-x). 